\title{
Improved time-domain accuracy standards for model gravitational waveforms
}

\author{
Lee Lindblom, ${ }^{1}$ John G. Baker, ${ }^{2}$ and Benjamin J. Owen ${ }^{3}$ \\ ${ }^{1}$ Theoretical Astrophysics 350-17, California Institute of Technology, Pasadena, California 91125, USA \\ ${ }^{2}$ Gravitational Astrophysics Laboratory, NASA/GSFC, Greenbelt, Maryland 20771, USA \\ ${ }^{3}$ Institute for Gravitation and the Cosmos, Center for Gravitational Wave Physics, Department of Physics, \\ The Pennsylvania State University, University Park, Pennsylvania 16802, USA
}

(Received 10 August 2010; published 12 October 2010)

\begin{abstract}
Model gravitational waveforms must be accurate enough to be useful for detection of signals and measurement of their parameters, so appropriate accuracy standards are needed. Yet these standards should not be unnecessarily restrictive, making them impractical for the numerical and analytical modelers to meet. The work of Lindblom, Owen, and Brown [Phys. Rev. D 78, 124020 (2008)] is extended by deriving new waveform accuracy standards which are significantly less restrictive while still ensuring the quality needed for gravitational-wave data analysis. These new standards are formulated as bounds on certain norms of the time-domain waveform errors, which makes it possible to enforce them in situations where frequency-domain errors may be difficult or impossible to estimate reliably. These standards are less restrictive by about a factor of 20 than the previously published time-domain standards for detection, and up to a factor of 60 for measurement. These new standards should therefore be much easier to use effectively.
\end{abstract}

DOI: $10.1103 /$ PhysRevD.82.084020

PACS numbers: 07.05.Kf, 04.25.D-, 04.25.dg, 04.30.-w

\section{INTRODUCTION}

Expected astrophysical sources of observable gravitational waves are often systems dominated by strong gravitational dynamics. In many cases this allows clear detailed predictions for the gravitational waveform signals, based on Einstein's theory, general relativity. While these theoretical predictions are well defined in principle, explicit computation of the model waveforms requires a combination of challenging analytic [1] and numerical [2] computations to approximate the ideal theoretical expectations. If the approximation errors are too large they will impact the interpretation of gravitational-wave data from operating gravitational-wave instruments such as LIGO [3] and Virgo [4], or future instruments such as LISA [5] and LCGT [6].

Model waveforms are used for matched filtering in gravitational-wave data analysis both for detection and for measurement of the physical parameters of the source (referred to as parameter estimation by the data analysis community). A signal is first identified in a detector's noisy data stream when it is found to have a significantly large correlation with (i.e. a sufficiently large projection onto) a model waveform. If the model waveforms were not accurate enough, then an unacceptably large fraction of real signals would fail to be detected in this way. The second use of model waveforms is to measure the physical properties of any signals identified in the detection step. These measurements are performed by fine-tuning the modelwaveform parameters (e.g., the masses and spins of the components of a compact binary, the source's orientation, the times of arrival of the signals, etc.) to achieve the largest correlation with the data. If the model waveforms were not accurate enough, these measured parameters would fail to represent the true physical properties of the sources to the level of precision commensurate with the intrinsic quality of the data. The systematic errors due to modeling inaccuracies should be no greater than the statistical errors due to detector noise. Model waveforms produced by numerical relativity simulations, e.g. of binary black holes, are computationally intensive to generate, and thus it is desirable to know what minimum accuracy standards are needed for these two uses. Flanagan and Hughes [7] made the first derivation of abstract and generic accuracy standards in this context. Miller [8] made the first concrete application of these standards to binary black-hole waveforms in numerical relativity, comparing post-Newtonian errors to thencurrent simulations and arguing for improvements in the latter. Our recent work has focused on finding simple intuitive derivations of the fundamental accuracy standards, extending the standards to include the effects of detector calibration errors, and deriving new representations of the standards that make it easier for the numerical relativity community to verify the waveform accuracy needed for gravitational-wave data analysis [9-11].

These accuracy standards are expressed as limits on the waveform-modeling errors $\delta h_{m}=h_{m}-h_{e}$, the difference between a model waveform, $h_{m}$, and its exact counterpart, $h_{e}$. Numerically modeled gravitational waveforms, and the errors associated with them, are generally evaluated most easily as functions of time, $h_{m}(t)$ and $\delta h_{m}(t)$. In contrast gravitational-wave data analysis and the accuracy standards for model waveforms are most conveniently formulated in terms of the frequency-domain representations of the waveforms and their errors, $h_{m}(f)$ and $\delta h_{m}(f)$. The time- and frequency-domain representations are related to one another by Fourier transforms, e.g., 


$$
h_{m}(f)=\int_{-\infty}^{\infty} h_{m}(t) e^{-2 \pi i f t} d t .
$$

So it is straightforward (in principle) to transform from one representation to the other.

The simplest way to express the standards needed to ensure the appropriate levels of accuracy for model gravitational waveforms is to write them in terms of a particular norm of the model-waveform errors: $\left\langle\delta h_{m} \mid \delta h_{m}\right\rangle$. This norm, defined by

$$
\left\langle\delta h_{m} \mid \delta h_{m}\right\rangle=4 \int_{0}^{\infty} \frac{\delta h_{m}(f) \delta h_{m}^{*}(f)}{S_{n}(f)} d f,
$$

weights the different frequency components of the waveform error by the power spectral density of the detector noise $S_{n}(f)$. In terms of this norm, the accuracy requirement that ensures no loss of scientific information during the measurement process is

$$
\sqrt{\frac{\left\langle\delta h_{m} \mid \delta h_{m}\right\rangle}{\left\langle h_{m} \mid h_{m}\right\rangle}}<\frac{\eta_{c}}{\rho}
$$

cf. Secs. VI and VII of Ref. [7] and Eq. (5) of Ref. [9]. Here $\rho=\sqrt{\left\langle h_{e} \mid h_{e}\right\rangle}$ is the optimal signal-to-noise ratio of the detected signal, and the parameter $\eta_{c} \lesssim 1$ is set by the level of calibration error in the detector, cf. Appendix A and Ref. [10]. Similarly the accuracy requirement that ensures no significant reduction in the rate of detections is

$$
\sqrt{\frac{\left\langle\delta h_{m} \mid \delta h_{m}\right\rangle}{\left\langle h_{m} \mid h_{m}\right\rangle}}<\sqrt{2 \epsilon_{\max }}
$$

where $\epsilon_{\max }$ is a parameter which determines the fraction of detections that are lost due to waveform-modeling errors, cf. Eq. (14) of Ref. [9]. The choice $\epsilon_{\max }=0.005$ ensures that no more than $10 \%$ of the signals will be missed, assuming the 0.03 mismatch used in the template bank discretization currently used in LIGO searches for coalescing compact binaries [9].

The basic accuracy standards, Eqs. (3) and (4), give the needed bounds on the waveform errors $\delta h_{m}$. Unfortunately, these abstract requirements are difficult, or perhaps impossible, to enforce directly in practice [11]. One fundamental problem is that an exact knowledge of the error, $\delta h_{m}$, associated with a model waveform, $h_{m}$, is never known. If $\delta h_{m}$ were known, then the exact waveform $h_{e}=h_{m}-\delta h_{m}$ would also be known, and there would be no need for accuracy standards. At best, the waveformmodeling community can aspire to construct tight upper bounds on these errors, $\left|\delta h_{m}\right| \leq \delta H_{m}$, that would make it possible to guarantee the needed waveform accuracy standards. Such bounds are often very difficult to construct. So in the absence of such bounds, good estimates of the errors, $\delta h_{m} \approx \delta H_{m}$, are a good way to verify that the accuracy standards are satisfied at least approximately.
Another (more practical) problem with the basic accuracy standards is that they are formulated in terms of the frequency-domain errors $\delta h_{m}(f)$. Since numerical model waveforms are generally computed in the time domain, $h_{m}(t)$, it will almost always be easier and more straightforward to bound or estimate the time-domain waveform errors $\delta h_{m}(t)$. Time-domain quantities are converted to the frequency domain via the Fourier transform, Eq. (1). In principle this is straightforward. In practice, however, it is easy to introduce errors into this process that can be larger than the intrinsic waveform-modeling errors. Discontinuities and other nonsmoothness at the beginning and end of a finite length waveform can create significant errors in the frequency-domain representation, unless appropriate "windowing" procedures are followed. This can be done (cf. Ref. [12] for a recent suggestion) but it requires some care and has some limitations on applicability. Also, more fundamentally, a time-domain bound does not translate to a frequency-domain bound [11]: that is, $\delta h_{m}(t)<\delta H_{m}(t)$ does not guarantee $\delta h_{m}(f)<$ $\delta H_{m}(f)$. For reasons of convenience and reliability therefore, it is very desirable (perhaps even necessary in some cases) to have versions of the accuracy standards formulated directly in terms of the time-domain waveform errors $\delta h_{m}(t)$. So this paper is devoted to finding time-domain representations of these standards that are straightforward and practical to use without being overly restrictive.

The most straightforward approach to obtaining the needed time-domain expression for the waveform accuracy would be to rewrite the fundamental noise-weighted norm $\left\langle\delta h_{m} \mid \delta h_{m}\right\rangle$ in terms of a suitably defined time-domain inner product. This can be done exactly using the "noise kernel" $k(t)$, defined as the real part of the Fourier transform of $2 S_{n}^{-1}(f)$. The resulting time-domain norm is then identical to the standard noise-weighted frequency-domain norm (e.g. Ref. [13]):

$$
\left\langle\delta h_{m} \mid \delta h_{m}\right\rangle=\int_{-\infty}^{\infty} \int_{-\infty}^{\infty} \delta h_{m}\left(t_{1}\right) k\left(t_{1}-t_{2}\right) \delta h_{m}\left(t_{2}\right) d t_{1} d t_{2} .
$$

Since this norm is identical to the standard noise-weighted norm, it can be substituted directly into the fundamental accuracy standards, Eqs. (3) and (4). Unfortunately for the same reason, it also suffers from both of the problems discussed above. Upper bounds in the time domain $\left|\delta h_{m}(t)\right| \leq \delta H_{m}(t)$ do not produce upper bounds on these time-domain norms. And errors in the norms introduced by Fourier transforms of finite duration waveforms with nonsmooth edges (Gibbs phenomena, etc.) also occur, because this time-domain norm is nonlocal in time on the time scales to which the detector is sensitive. These problems could be avoided if there were efficient representations of the accuracy standards that placed limits on local-in-time norms of the waveform errors. The purpose of this paper is 
to construct new formulations of the waveform accuracy standards that meet these criteria.

Before moving on to a detailed discussion of timedomain error standards themselves, it is worth pausing to think briefly about how waveform errors $\delta h_{m}$ have been (or could be) bounded or estimated. Error bounds (called $a$ posteriori error estimates in the mathematics literature) have been constructed for quantities derived from the numerical solutions of hyperbolic evolution problems [14]. However no results of this kind are known at this time (to our knowledge) specifically for the case of gravitational waveforms extracted from solutions of Einstein's equations. The error bounds that do exist are for timedomain errors, and we know of no frequency-domain bounds for quantities derived from the solutions of timeevolution problems at all. Given a time-domain error bound, $\delta H_{m}(t)$, it would only be useful for enforcing the basic accuracy standards if it could be converted to a frequency-domain bound $\left|\delta h_{m}(f)\right| \leq \delta H_{m}(f)$, or if a time-domain version of the accuracy standards were available. Unfortunately the Fourier transform of $\delta H_{m}(t)$ does not generally provide the needed frequency-domain bound, since $\left\langle\delta H_{m} \mid \delta H_{m}\right\rangle$ is not always larger than $\left\langle\delta h_{m} \mid \delta h_{m}\right\rangle$ [11]. Time-domain bounds, $\delta H_{m}(t)$, are only useful therefore if there are versions of the basic accuracy standards, Eqs. (3) and (4), based on local-in-time norms of the waveform error.

Good approximations of the waveform errors $\delta h_{m}(t) \approx$ $\delta H_{m}$ can be constructed using Richardson extrapolation methods. Model waveforms generally have errors that scale with some accuracy parameter, $a$, in a wellunderstood way. ${ }^{1}$ Many methods of computing waveforms have dominant errors that scale as some power of this parameter, $\delta h_{m}(t) \approx A(t) a^{n}$, while others have errors that scale exponentially, $\delta h_{m}(t) \approx A(t) e^{-\alpha / a}$. In either case the values of the quantities $A(t), n, \alpha$, etc. can be evaluated by comparing the waveforms $h_{m}\left(t, a_{i}\right)$ produced by simulations using different values of $a_{i}$. The errors in the most accurate of these simulations, $a=a_{\min }$, are then given approximately by the scaling expression, e.g., $\delta h_{m}(t) \approx$ $\delta H_{m}(t)=A(t) a_{\min }^{n}$, using the measured values of the various constants. These Richardson extrapolation methods could also be applied to frequency-domain representations of the waveforms evaluated at different accuracies, $h_{m}\left(f, a_{i}\right)$ (subject to the problems involved in performing the Fourier transforms discussed above). The resulting frequency-domain error estimates, e.g., $\delta h_{m}(f) \approx$ $\delta H_{m}(f)=A(f) a_{\min }^{n}$, could then be used directly to estimate whether the fundamental accuracy standards are satisfied. The existence of accuracy standards based on localin-time norms just provides a more convenient and perhaps

\footnotetext{
${ }^{1}$ This parameter could represent the spacing between grid points for numerical waveform calculations, or perhaps a postNewtonian-like expansion parameter for an analytic calculation.
}

a more reliable way to evaluate waveform accuracy in this case, rather than being a necessity as it was in the errorbound case. The gravitational waveform-simulation community has begun to estimate waveform errors by comparing time-domain waveforms computed with different accuracy parameters [15-22]. However, Richardson extrapolation methods have not yet been applied to improve the quality of these error estimates (to our knowledge).

The discussion above shows that alternate versions of the basic accuracy standards, based on local-in-time norms of the waveform errors, are needed for reasons of convenience and perhaps necessity. This paper constructs a number of new versions of the standards that meet all the needed criteria. Some representations of the accuracy standards based on local-in-time norms of the time-domain errors have already been discussed in the literature $[9,11]$. While these are sufficient to guarantee the fundamental accuracy requirements, Eqs. (3) and (4), they achieve this at the price of placing excessive restrictions on the allowed time-domain errors. New time-domain accuracy standards are developed here that are considerably less restrictive: about a factor of 20 for the detection standards, and up to a factor of 60 for measurement. These new standards are at most a factor of 3 more restrictive than their optimal frequency-domain counterparts, so we expect they should be practical to use. The previous work on timedomain accuracy standards is reviewed, and the new standards are derived and discussed in detail in the following sections of this paper.

\section{TIME-DOMAIN ACCURACY STANDARDS}

The fundamental frequency-domain accuracy standards, Eqs. (3) and (4), can also be expressed in terms of the timedomain $L^{2}$ norm of the waveform errors $[9,11]$. These time-domain versions provide a way to enforce the waveform-accuracy standards without the need for a detailed knowledge of the frequency-domain representation of the errors. This section reviews the derivation of the presently known time-domain standards, and illustrates the shortcomings that make them an impractical way to enforce the accuracy requirements. The methods of analysis used to derive the original time-domain standards are then generalized to produce several new and improved versions. Each of these new standards is itself a sufficient condition that can be used to enforce the fundamental frequencydomain standards. As an illustration, these new standards are applied to the case of binary black-hole inspiralmerger-ringdown waveforms for a detector with an Advanced LIGO noise curve [23]. This example shows the effectiveness and utility of these new time-domain standards as tools for waveform accuracy enforcement.

The frequency-domain accuracy standards, which are based on the noise-weighted norm $\left\langle\delta h_{m} \mid \delta h_{m}\right\rangle$, can be converted to time-domain standards using the following basic inequality: 


$$
\left\langle\delta h_{m} \mid \delta h_{m}\right\rangle \leq \frac{4 \int_{0}^{\infty}\left|\delta h_{m}(f)\right|^{2} d f}{\min \left[S_{n}(f)\right]} .
$$

This inequality approximates the power spectral density of the detector noise as a constant: its minimum value. The approximation in Eq. (6) is very good therefore whenever the model-waveform error is largest in the sensitive band of the detector, and is not so good when a significant part of the error lies outside this sensitive band. By Parseval's theorem, the numerator in Eq. (6) is proportional to the time-domain $L^{2}$ norm, $\left\|\delta h_{m}(t)\right\|$ :

$$
\left\|\delta h_{m}(t)\right\|^{2} \equiv \int_{-\infty}^{\infty}\left|\delta h_{m}(t)\right|^{2} d t=2 \int_{0}^{\infty}\left|\delta h_{m}(f)\right|^{2} d f
$$

Thus the basic inequality, Eq. (6), can be rewritten as

$$
\left\langle\delta h_{m} \mid \delta h_{m}\right\rangle \leq \frac{2\left\|\delta h_{m}(t)\right\|^{2}}{\min \left[S_{n}(f)\right]} .
$$

Using this inequality, the fundamental accuracy standards, Eqs. (3) and (4), can be rewritten in terms of time-domain $L^{2}$ norms:

$$
\frac{\left\|\delta h_{m}(t)\right\|}{\left\|h_{m}(t)\right\|} \leq C_{0} \frac{\eta_{c}}{\rho}
$$

for measurement, and

$$
\frac{\left\|\delta h_{m}(t)\right\|}{\left\|h_{m}(t)\right\|} \leq C_{0} \sqrt{2 \epsilon_{\max }}
$$

for detection. The quantity $C_{0}$ that appears in these expressions is defined as the ratio of the traditional optimal signal-to-noise measure $\rho$ to a nontraditional measure:

$$
C_{0}^{2}=\rho^{2}\left\{\frac{2\left\|h_{m}(t)\right\|^{2}}{\min \left[S_{n}(f)\right]}\right\}^{-1} .
$$

This quantity is dimensionless and independent of the overall scale (i.e. the distance to the source) used in $h_{m}$.

The time-domain standards, Eqs. (9) and (10), differ therefore from the fundamental standards, Eqs. (3) and (4), in just two ways: (i) the norms used to measure the waveform errors on the left sides are different, and (ii) the maximum allowed errors on the right sides are multiplied by the factor $C_{0}$ in the time-domain case. It is straightforward to show that $C_{0}$ is always less than 1: $C_{0} \leq 1$. Thus the time-domain standards are always more restrictive than their frequency-domain counterparts. The quantity $C_{0}$ compensates in the time-domain standards for the fact that the waveform errors are not being weighted by the detector noise in the optimal way. Unfortunately these time-domain standards have two serious flaws: The first is that the time-domain norms of the exact waveforms $\left\|h_{e}(t)\right\|$ are not well defined, because the waveforms $h_{e}(t)$ do not fall to zero quickly enough as $t \rightarrow-\infty$. Therefore the norms of the model waveforms $\left\|h_{m}(t)\right\|$, and thus the quantity $C_{0}$, are influenced (although fairly weakly) by an unphysical parameter, the length of the simulation. The second flaw, discussed in more detail below, is that $C_{0}$ is quite small for realistic waveforms, thus making these time-domain versions of the standards very overrestrictive.

To determine how overly restrictive the time-domain standards are in practice, the quantity $C_{0}$ has been evaluated for nonspinning equal-mass black-hole binary waveforms and for a detector with an Advanced LIGO noise curve optimized for double neutron-star binaries [23]. ${ }^{2}$ These waveforms were constructed by patching together post-Newtonian waveforms for the early inspiral phase, with numerical relativity waveforms for the late inspiral plus merger plus ringdown phases [24,25] and adjusting the time scales for different total masses. They are the same used earlier to evaluate $C_{0}$ [9]. Figure 1 illustrates $C_{0}$ as a function of mass for binary black-hole systems with masses in the range that is relevant for Advanced LIGO: $4 \leq M / M_{\odot} \leq 400$. For low-mass binary systems-where these model waveforms lie mostly within the Advanced LIGO frequency band $-C_{0}$ is of order unity, and the timedomain standards are reasonably efficient representations of the fundamental frequency-domain standards. However, for binary systems with large masses-where these model waveforms lie mostly outside the Advanced LIGO band$C_{0}$ becomes very small, and the time-domain standards become very ineffective. For the largest masses the quantity $C_{0}$ becomes very small, $C_{0} \approx 0.016$, and the timedomain standards must place unreasonably tight constraints on the waveform errors to ensure that the fundamental frequency-domain standards are satisfied by the small part of these waveforms lying within the Advanced LIGO band.

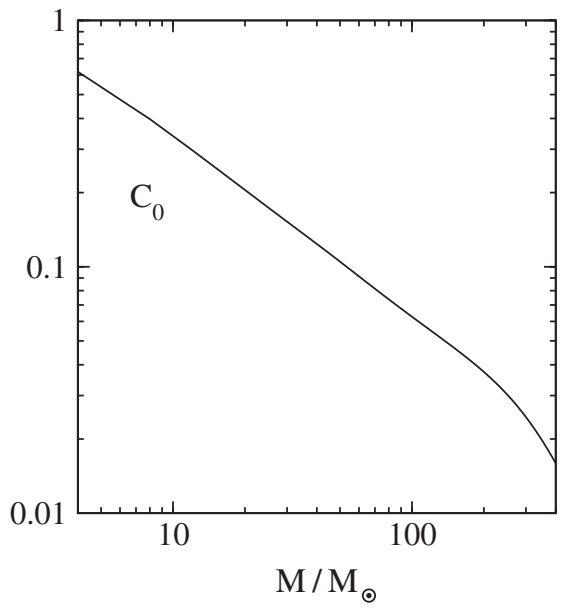

FIG. 1. Curve illustrates $C_{0}$, the ratio of the traditional optimal signal-to-noise measure $\rho$ to a nontraditional measure defined in Eq. (11), as a function of the total mass for nonspinning equalmass black-hole binary waveforms and for a detector with an Advanced LIGO noise curve optimized for double neutron-star binaries. 
The methods of analysis used to derive Eqs. (9) and (10) can be generalized to obtain a new set of time-domain accuracy standards. This can be done using the following extension of the basic inequality:

$$
\left\langle\delta h_{m} \mid \delta h_{m}\right\rangle \leq \frac{4 \int_{0}^{\infty}(2 \pi f)^{2 k}\left|\delta h_{m}(f)\right|^{2} d f}{\min \left[(2 \pi f)^{2 k} S_{n}(f)\right]} .
$$

The numerator in this expression is just the frequencydomain $L^{2}$ norm of the $k$ th time derivative of the waveform error. This can be converted to a time-domain $L^{2}$ norm using Parseval's theorem:

$$
\begin{aligned}
\left\|\delta h_{m}(t)\right\|_{k}^{2} & \equiv \int_{-\infty}^{\infty}\left|\frac{d^{k} \delta h_{m}(t)}{d t^{k}}\right|^{2} d t, \\
& =2 \int_{0}^{\infty}(2 \pi f)^{2 k}\left|\delta h_{m}(f)\right|^{2} d f .
\end{aligned}
$$

The basic inequality in Eq. (12) can therefore be rewritten as

$$
\left\langle\delta h_{m} \mid \delta h_{m}\right\rangle \leq \frac{2\left\|\delta h_{m}(t)\right\|_{k}^{2}}{\min \left[(2 \pi f)^{2 k} S_{n}(f)\right]} .
$$

These inequalities together with the fundamental frequency-domain accuracy standards, Eqs. (3) and (4), can then be used to obtain a new set of time-domain accuracy standards:

$$
\mathcal{E}_{k} \leq C_{k} \frac{\eta_{c}}{\rho},
$$

for measurement, and

$$
\mathcal{E}_{k} \leq C_{k} \sqrt{2 \epsilon_{\max }}
$$

for detection. The quantities $\mathcal{E}_{k}$,

$$
\mathcal{E}_{k} \equiv \frac{\left\|\delta h_{m}(t)\right\|_{k}}{\left\|h_{m}(t)\right\|_{k}}
$$

are new measures of the time-domain waveform error, and the quantities $C_{k}$ are defined as the ratios of the traditional optimal signal-to-noise measure $\rho$ to new nontraditional measures:

$$
C_{k}^{2}=\rho^{2}\left\{\frac{2\left\|h_{m}(t)\right\|_{k}^{2}}{\min \left[(2 \pi f)^{2 k} S_{n}(f)\right]}\right\}^{-1} .
$$

Both quantities, $\mathcal{E}_{k}$ and $C_{k}$, are dimensionless and independent of the overall scale (i.e. the distance to the source) used in $h_{m}$. It is straightforward to show that $C_{k} \leq 1$, so these new standards are always more restrictive than their frequency-domain counterparts. The $k=0$ versions of Eqs. (15) and (16) are identical to the previously known time-domain standards, Eqs. (9) and (10). But the

\footnotetext{
${ }^{2}$ The data for this neutron-star optimized noise curve come from the file NSNS_Opt.txt available from the URL in Ref. [23].
}

standards with $k \geq 1$ are new and distinct. The new standards with $k \geq 1$ are free from the flaws described earlier for the previously known $k=0$ standards. The norms $\left\|h_{m}(t)\right\|_{k}$ are well defined for $k \geq 1$, because $d^{k} h_{m} / d t^{k}$ falls off quickly enough as $t \rightarrow-\infty$. And as described in more detail below, the $C_{k}$ for $k \geq 1$ are much closer to 1 . So these new standards are much more effective ways to enforce the accuracy requirements.

Figure 2 illustrates the quantities $C_{k}$ (for $k=0,1$, and 2) that appear in the waveform accuracy standards, Eqs. (15) and (16), as a function of the mass of the waveform. Each of these new accuracy standards is sufficient to guarantee that the corresponding frequency-domain standard is satisfied. Choosing the easiest standard to satisfy is all that is required for a given $M$, and this will typically be the one having the largest $C_{k}$. Figure 2 shows that the standards with different values of $k$ have different mass ranges where they are most effective. The $k=0$ standard is most effective for low-mass binary waveforms, while the $k=2$ standard is the most effective for high-mass waveforms.

Figure 3 shows the noise curves used to compute the $C_{k}$ illustrated in Fig. 2. These particular noise curves are all representations of the Advanced LIGO sensitivity curves optimized for neutron-star/neutron-star binary signals [23]. The noise curves with successively larger values of $k$ have minima at successively smaller values of the frequency $f$. Figure 3 shows that the factor $f^{2 k}$ combines with the piecewise power law structure of $S_{n}(f)$ to produce fairly broad regions where the effective noise curves are rather flat. In these flat regions the approximation, $\min \left[f^{2 k} S_{n}(f)\right] \approx f^{2 k} S_{n}(f)$, becomes extremely good, and the approximation leading to Eq. (12) becomes nearly

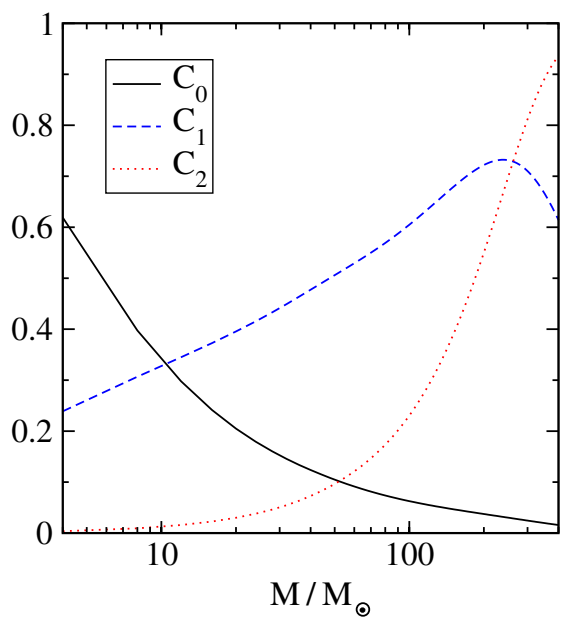

FIG. 2 (color online). Curves illustrate $C_{k}$, the ratio of the traditional optimal signal-to-noise measure $\rho$ to a nontraditional measure defined in Eq. (18), as a function of the total mass for nonspinning equal-mass black-hole binary waveforms. The waveform accuracy standards, Eqs. (15) and (16), can be enforced using any value of $k$. Thus it is sufficient to impose the standard having the largest $C_{k}$ in each mass range. 


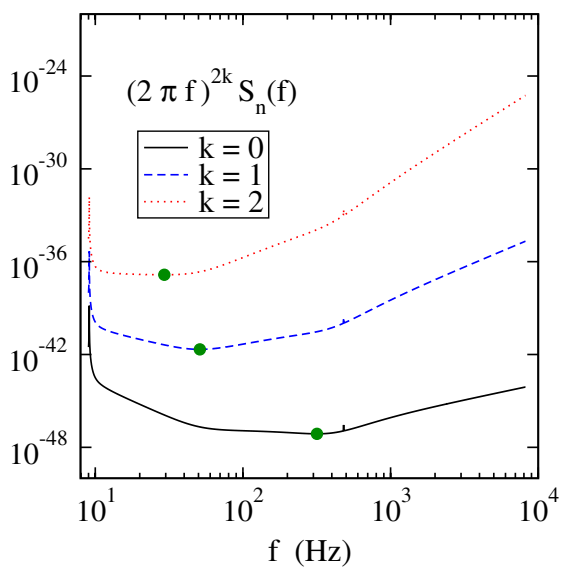

FIG. 3 (color online). Curves illustrate the effective noise curves, $(2 \pi f)^{2 k} S_{n}(f)$, that appear in the norms of the $k$ th time derivatives of the waveform. The minima of these curves are marked by the large dots. These curves illustrate why the higher time-derivative norms are more sensitive to the lower-frequency parts of the waveforms, and hence do a better job of modeling the waveform accuracy in high-mass binaries.

exact. These features explain one of the reasons the higher time-derivative norms measure the lower-frequency parts of the waveforms more faithfully, and hence do a better job of enforcing the waveform accuracy standards in highmass waveforms. The higher time-derivative norms measure $d^{k} h / d t^{k}$, which is more strongly peaked near the time of merger. Hence the energy norms $\mathcal{E}_{k}$ for $k \geq 1$ do a better job than $\mathcal{E}_{0}$ of measuring the strongest parts of the waveform that contribute most to detection.

Model waveforms used to measure accurately the physical parameters of a previously detected signal may be custom made for this task. The physical parameters of the waveform will be known (approximately) in this case from the detection process, so a finely targeted template bank of waveforms with parameters that cover a small neighborhood surrounding the actual physical values can be computed. For such applications, the quantities $C_{k}$ can easily be computed along with the model waveforms. The waveform accuracy standard for measurement, Eq. (15), should be enforced therefore using the standard having the largest $C_{k}$ for these particular waveforms. As Fig. 2 shows, this will typically be the $k=2$ standard for the largest mass binary waveforms, $k=0$ for the smallest mass waveforms, and $k=1$ for a broad range of intermediate mass waveforms. The smallest value of $C_{k}$ needed for these measurement tests is $C_{0}=C_{1} \approx 0.331$ if the mass of the waveform is near $10 M_{\odot}$. However, if the mass is larger, the value of the $C_{k}$ could be as large as $C_{2} \approx 0.935$ for waveforms with masses near $400 M_{\odot}$. These new time-domain accuracy standards for measurement can therefore be up to 60 times less restrictive (depending on the mass of the waveform) than the previously available $k=0$ standard [9].
The situation is somewhat more complicated for waveforms used as part of the detection process. These waveforms should be scalable to the full range of masses whose waveforms lie within the sensitivity band of the detector. The simplest way to do this would be to use the accuracy standard having the largest $\min _{M} C_{k}(M)$, where the minimization is done over the waveform masses in the appropriate range. Note that the quantity $\mathcal{E}_{k}$, which appears on the left side of Eq. (16), is independent of mass because both $\left\|\delta h_{m}\right\|_{k}$ and $\left\|h_{m}\right\|_{k}$ scale with mass in exactly the same way. Thus the accuracy standard for a given $k$ can be enforced over the entire range of masses by ensuring that $\mathcal{E}_{k}$ satisfies the standard with $C_{k}=\min _{M} C_{k}(M)$. The $k=$ 1 standard has the largest $\min _{M} C_{k}(M)$ for the waveforms illustrated in Fig. 2, so it is sufficient in this case to enforce the $k=1$ standard with $C_{1}=\min _{M} C_{1}(M) \approx 0.239$. This is about 15 times less restrictive than would be required using the previously available $k=0$ standard where $\min _{M} C_{0}(M) \approx 0.016$.

A less restrictive accuracy standard for detection can also be constructed, at the expense of making it slightly more complicated. Figure 2 shows that enforcing the detection standard, Eq. (16), with $C_{1}=\min _{M} C_{1}(M)$ is somewhat more restrictive than is really necessary. All that is required is to enforce the standard having the largest $C_{k}(M)$ for each mass. So it would be sufficient to enforce the $k=$ 0 standard in the mass range where $C_{0}$ is the largest of the $C_{k}: M \leq M_{\text {int }}$. The mass $M_{\text {int }} \approx 10.4 M_{\odot}$ represents the point where the $C_{0}(M)$ and the $C_{1}(M)$ curves intersect: $C_{0}\left(M_{\text {int }}\right)=C_{1}\left(M_{\text {int }}\right) \approx 0.331$ for the waveform example shown in Fig. 2. It would also be sufficient to enforce the $k=1$ standard in the mass range $M \geq M_{\text {int }}$. Therefore it is sufficient over the full range of masses to enforce both the $k=0$ and the $k=1$ versions of the detection accuracy standard, Eq. (16), with $C_{0}\left(M_{\text {int }}\right)=C_{1}\left(M_{\text {int }}\right) \approx 0.331^{3}$ :

$$
\mathcal{E}_{0} \lesssim 0.331 \sqrt{2 \epsilon_{\max }}, \quad \text { and } \quad \mathcal{E}_{1} \lesssim 0.331 \sqrt{2 \epsilon_{\max }} \text {. }
$$

This condition is about 20 times less restrictive than would be required to enforce the previously available $k=0$ standard over the full range of masses. The overall factor that appears on the right sides in Eq. (19) appears to be quite insensitive to the detector noise curve, giving 0.351 instead of 0.331 using the "zero-detuning, high-power" version of the Advanced LIGO noise curve. ${ }^{4}$ We have not yet determined how sensitive this factor is to properties of the waveform models, like the total duration of the waveform, or the spins of the individual black holes.

\footnotetext{
${ }^{3}$ In principle it would be sufficient to enforce the $k=2$ instead of the $k=1$ standard in the mass range $M \gtrsim 260 M_{\odot}$. However, this would not make the needed $k=1$ condition any weaker, because $\mathcal{E}_{1}$ is independent of mass.

${ }^{4}$ The data for this zero-detuning, high-power noise curve comes from the file ZERO_DET_high_P.txt available from the URL in Ref. [23].
} 


\section{DISCUSSION}

New time-domain representations of the waveform accuracy standards for gravitational-wave data analysis have been presented in Sec. II. For waveforms to be used for measuring the physical properties of a detected signal, the new time-domain standards, Eq. (15), provide the best way to enforce the needed accuracy standards. These standards give the allowed upper limits on certain new measures of the waveform error $\mathcal{E}_{k}$, defined in terms of the time-domain $L^{2}$ norms of the $k$ th time derivatives of the model waveform $h_{m}$ and its error $\delta h_{m}: \mathcal{E}_{k} \equiv\left\|\delta h_{m}(t)\right\|_{k} /\left\|h_{m}(t)\right\|_{k}$. Model waveforms are generally obtained by performing time integrals of an equation like $d^{2} h_{m}(t) / d t^{2}=\tilde{\Psi}_{4}(t)$, where $\tilde{\Psi}_{4}(t)$ is the (real) projection of the outgoing component of the Weyl curvature representing the wave polarization of interest. Therefore, it is no harder or less accurate to evaluate the first two time derivatives of $h_{m}(t)$, and their accuracy, than it is to evaluate $h_{m}(t)$ and its accuracy. For the binary blackhole waveforms used as an example here, the $k=0$ version of this standard is most effective for black-hole systems with masses below about $10 M_{\odot}$; the $k=1$ standard is best in the intermediate mass range $10 \leqq M / M_{\odot} \lesssim 260$; and the $k=2$ standard is best for the highest mass waveforms, $260 \leqq M / M_{\odot} \leq 400$. This new version of the accuracy standard for measurement is less restrictive than the previously available time-domain standard by up to a factor of 60 for the largest mass binary systems, and is never more restrictive than the ideal frequency-domain standards by a factor greater than about 3 . The new standard for measurement is nevertheless sufficient to ensure that the ideal frequency-domain standard is satisfied as well.

The situation is slightly more complicated for the new time-domain waveform accuracy standards for detection. These standards put limits on both the $k=0$ and $k=1$ versions of the time-domain error measures, $\mathcal{E}_{0}$ and $\mathcal{E}_{1}$. The best new time-domain standard for detection presented in Sec. II enforces these standards with $C_{0}=C_{1} \approx 0.331$ for the example waveform used here. This new standard for detection, Eq. (19), guarantees that the frequency-domain standards are enforced, and it is only about a factor of 3 more restrictive than those ideal standards. The new standards should be effective enough therefore to make them usable without placing an undue burden on the waveformsimulation community. Slightly less restrictive timedomain standards for detections can also be derived using methods similar to those used in Sec. II. The derivation of these additional alternative standards is discussed and analyzed in detail in Appendix B. These alternative standards are more complicated, however, and consequently may not prove to be as useful as Eq. (19).

\section{ACKNOWLEDGMENTS}

We thank Michael Holst for valuable discussions about rigorous mathematical error bounds, Sean McWilliams for other useful discussions, and Duncan Brown for helpful comments on an earlier draft of this paper. This research was supported in part by grants to Caltech from the Sherman Fairchild Foundation, NSF Grants No. DMS0553302, No. PHY-0601459, and No. PHY-0652995, and NASA Grant No. NNX09AF97G; by NASA Grants No. 08-ATFP08-0126 and No. 09-ATP09-0136 to Goddard Space Flight Center; by NSF Grant No. PHY0855589 to Penn State; and by the LIGO Visitors Program. LIGO was constructed by the California Institute of Technology and Massachusetts Institute of Technology with funding from the National Science Foundation and operates under cooperative agreement PHY-0757058. This paper has LIGO Document No. LIGO-P1000078.

\section{APPENDIX A: CALIBRATION ERROR LEVELS}

The basic accuracy requirement for measurement, Eq. (3), reduces to the case of an ideal detector when $\eta_{c}=$ 1. The ideal-detector approximation means that the systematic errors made in calibrating the detector (by measuring the response function of the detector) are negligible compared to the statistical errors due to the intrinsic noise level of the detector. It is more realistic to expect that in many cases the detector will have non-negligible calibration errors. These errors influence the data analysis process in almost the same way as waveform-modeling errors [10]. Scientific information will be lost unless the combined systematic calibration and waveform-modeling errors are smaller than the intrinsic detector noise level. This requires that both the calibration error and the waveform-modeling errors be kept below the intrinsic noise level of the detector. The allowed error budget may however be apportioned arbitrarily between the two sources, e.g., in the most economical or convenient way. The parameter $\eta_{c}$ introduced in Eq. (3) determines the share of this error budget allowed for the waveform-modeling error. In particular it represents the amount by which the waveform-modeling error must be reduced below the intrinsic detector noise level of the detector to allow for the presence of calibration error.

In some cases detectors may have calibration errors that are negligible compared to the needed waveform-modeling errors (e.g., the proposed Laser Interferometer Space Antenna LISA). In those cases the ideal-detector conditions apply and $\eta_{c}=1$ is the appropriate choice. In most cases, however, it may only be possible or economically feasible to calibrate to an accuracy level comparable to the intrinsic detector noise. In these cases it seems likely that it will be optimal to split the available error budget equally between the calibration and waveform-modeling errors. If these errors can be considered uncorrelated, then $\eta_{c}=$ $1 / \sqrt{2}$ is the appropriate choice. Waveform-modeling errors, $\delta h_{m}$, are uncorrelated with the calibration errors, $\delta h_{R}$, if $\left\langle\delta h_{m} \mid \delta h_{R}\right\rangle^{2} \ll\left\langle\delta h_{m} \mid \delta h_{m}\right\rangle\left\langle\delta h_{R} \mid \delta h_{R}\right\rangle$. To justify taking $\eta_{c}=1 / \sqrt{2}$, this uncorrelated condition should be satisfied for all times when data are being collected, and for 
all the different errors associated with the various model waveforms that will be used in the subsequent data analysis. While calibration and modeling errors come from completely unrelated sources, it may be difficult to completely rule out the possibility of significant correlations. For example, it is easy to imagine both calibration and modeling errors that are proportional to the exact waveform $h_{e}$. When correlations cannot be ruled out, the somewhat stricter condition, $\eta_{c}=\frac{1}{2}$, is required. The calibration accuracy needed to ensure no loss of scientific information for the strongest sources is generally much greater than the accuracy needed for detection. The weak dependence of the detection standard on $\eta_{c}$ has therefore been ignored here, and the ideal-detector version of the standards, Eq. (4), are used in this paper.

\section{APPENDIX B: COMPOUND ACCURACY STANDARDS}

A somewhat more general class of time-domain accuracy standards are derived in this Appendix using the methods developed in Sec. II. These additional standards are somewhat less restrictive for detection than Eq. (19), but they are also more complex. The idea is to construct a new measure of the waveform error that uses a combination of the time-domain norms used in Eq. (16). This is done by splitting the integral that appears in the basic inequality, Eq. (12), into parts proportional to $\mu$ and $1-$ $\mu$ respectively (where $\mu$ is an arbitrary splitting factor that satisfies $0 \leq \mu \leq 1$ ), and then applying the constant-noise approximation using different values of $k$ for each part. The result is a compound version of the basic inequality:

$$
\begin{aligned}
\left\langle\delta h_{m} \mid \delta h_{m}\right\rangle \leq & \frac{4 \mu \int_{0}^{\infty}(2 \pi f)^{2 k}\left|\delta h_{m}(f)\right|^{2} d f}{\min \left[(2 \pi f)^{2 k} S_{n}(f)\right]} \\
& +\frac{4(1-\mu) \int_{0}^{\infty}(2 \pi f)^{2 k^{\prime}}\left|\delta h_{m}(f)\right|^{2} d f}{\min \left[(2 \pi f)^{2 k^{\prime}} S_{n}(f)\right]} .
\end{aligned}
$$

The right side of this inequality can be rewritten in terms of the time-domain error measures $\mathcal{E}_{k}$ of Eq. (17), and the quantities $C_{k}$ of Eq. (18):

$$
\frac{\left\langle\delta h_{m} \mid \delta h_{m}\right\rangle}{\left\langle h_{m} \mid h_{m}\right\rangle} \leq \frac{\mu \mathcal{E}_{k}^{2}}{C_{k}^{2}}+\frac{(1-\mu) \mathcal{E}_{k^{\prime}}^{2}}{C_{k^{\prime}}^{2}}
$$

This basic inequality can be transformed into a more compact and useful form by replacing the splitting parameter $\mu$ with a new parameter $\sigma$, defined by

$$
\mu=\frac{\sigma C_{k}^{2}}{\sigma C_{k}^{2}+(1-\sigma) C_{k^{\prime}}^{2}} .
$$

The parameter $\mu$ satisfies the required inequality, $0 \leq \mu \leq$ 1 , whenever $0 \leq \sigma \leq 1$. The basic inequality then becomes

$$
\frac{\left\langle\delta h_{m} \mid \delta h_{m}\right\rangle}{\left\langle h_{m} \mid h_{m}\right\rangle} \leq \frac{\mathcal{E}_{k, k^{\prime}}^{2}(\sigma)}{C_{k, k^{\prime}}^{2}(\sigma)},
$$

where $\mathcal{E}_{k, k^{\prime}}(\sigma)$ is a composite measure of the waveform error, defined by

$$
\mathcal{E}_{k, k^{\prime}}^{2}(\sigma)=\sigma \mathcal{E}_{k}^{2}+(1-\sigma) \mathcal{E}_{k^{\prime}}^{2},
$$

and $C_{k, k^{\prime}}(\sigma)$ is the composite analog of $C_{k}$, defined by

$$
C_{k, k^{\prime}}^{2}(\sigma)=\sigma C_{k}^{2}+(1-\sigma) C_{k^{\prime}}^{2} .
$$

This new form of the basic inequality, Eq. (B4), is true for any $\sigma$. It can be used to convert the fundamental frequency-domain standards, Eqs. (3) and (4), into new time-domain waveform accuracy standards:

$$
\mathcal{E}_{k, k^{\prime}}(\sigma) \leq C_{k, k^{\prime}}(\sigma) \frac{\eta_{c}}{\rho},
$$

for measurement, and

$$
\mathcal{E}_{k, k^{\prime}}(\sigma) \leq C_{k, k^{\prime}}(\sigma) \sqrt{2 \epsilon_{\max }},
$$

for detection. The waveform error measure $\mathcal{E}_{k, k^{\prime}}(\sigma)$ is positive definite for all $\sigma$ in the range $0 \leq \sigma \leq 1$. So these new time-domain waveform accuracy standards, Eqs. (B7) and (B8), are sufficient to enforce the fundamental accuracy standards for any $\sigma$ in this range. In the extreme cases $\sigma=0$ and $\sigma=1, \mathcal{E}_{k, k^{\prime}}(\sigma)$ reduces to one of the single $k$ waveform error measures and these standards reduce to special cases of Eqs. (15) and (16). For intermediate values $0<\sigma<1$ however, these time-domain waveform accuracy standards are completely new and distinct.

Figure 4 illustrates $C_{0,1}(\sigma)=\sqrt{\sigma C_{0}^{2}+(1-\sigma) C_{1}^{2}}$ as a function of mass for the waveform and noise curve used in the previous examples. These graphs show that $C_{0,1}(\sigma)$ reduces to $C_{0,1}(0)=C_{1}$ or $C_{0,1}(1)=C_{0}$ in the limiting

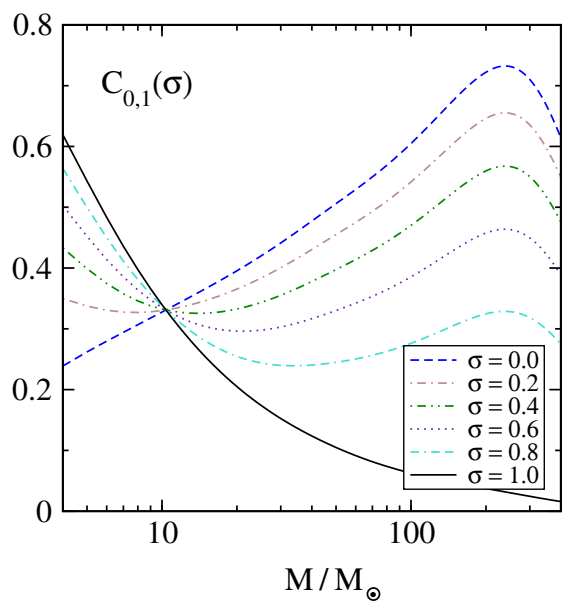

FIG. 4 (color online). Curves show $C_{0,1}(\sigma)=$ $\sqrt{\sigma C_{0}^{2}(M)+(1-\sigma) C_{1}^{2}(M)}$ as a function of mass for several values of the parameter $\sigma$. 
cases $\sigma=0$ or $\sigma=1$, respectively. These graphs also show that none of the $C_{0,1}(\sigma)$ is larger than one of the limiting curves, $\sigma=0$ or $\sigma=1$, for any value of $M$. Consequently the new compound accuracy standards for measurement, Eq. (B7), are generally more restrictive than the simpler single $k$ standard for measurement, Eq. (15). Therefore, the new compound accuracy standards are probably only useful for detection.

For detection, Eq. (B8) determines the maximum allowed sizes of the error measures $\mathcal{E}_{k, k^{\prime}}(\sigma)$. Since these measures are independent of the mass of the waveform, it follows that these detection standards will be satisfied for the full range of masses only if they are satisfied for $C_{k, k^{\prime}}(\sigma)=\min _{M} C_{k, k^{\prime}}(\sigma, M)$, where the minimization is over the relevant range of masses. Figure 4 shows that these minima depend on the parameter $\sigma$. Perhaps the simplest of these compound error measures is the one with $\sigma=0.5$, in which the two single $k$ error measures, $\mathcal{E}_{k}$ and $\mathcal{E}_{k^{\prime}}$, are weighted equally. For the waveforms used in this example, $\min _{M} C_{0,1}(0.5, M) \approx 0.314$, so the detection standard becomes $\mathcal{E}_{0,1}(0.5) \leq 0.314 \sqrt{2 \epsilon_{\max }}$. Since the factor 0.314 is a little smaller than the factor 0.331 that appears in Eq. (19), this particular standard is considered a little more restrictive. A deeper analysis below however shows that this compound standard is more restrictive in some cases but less restrictive in others compared to Eq. (19).

There does exist, however, an unconditionally less restrictive compound error standard for a particular optimal choice of the parameter $\sigma=\sigma_{\mathrm{opt}}$. Figure 4 shows that all the different $C_{0,1}(\sigma)$ curves intersect at the single point $M=M_{\text {int }}$ where $C_{0}\left(M_{\text {int }}\right)=C_{1}\left(M_{\text {int }}\right)$. Thus the largest of the $\min _{M} C_{0,1}(\sigma, M)$ can never be larger than $C_{0}\left(M_{\text {int }}\right)$. The optimal value $\sigma_{\text {opt }}$, for which $\min _{M} C_{0,1}\left(\sigma_{\text {opt }}, M\right)=$ $C_{0}\left(M_{\text {int }}\right)$, can be determined by enforcing the extremum condition, $d C_{0,1}^{2}(\sigma, M) / d M=0$, at the point where $M=M_{\text {int }}$. The result is

$$
\sigma_{\mathrm{opt}}=\frac{d C_{1}^{2}}{d M}\left(\frac{d C_{1}^{2}}{d M}-\frac{d C_{0}^{2}}{d M}\right)^{-1} \approx 0.284
$$

for the waveform and noise curve example discussed in Sec. II. The optimal version of the new compound timedomain accuracy standard for detection then becomes

$$
\mathcal{E}_{0,1}(0.284) \lesssim 0.331 \sqrt{2 \epsilon_{\max }} .
$$

This new version of the detection standard uses the value $C_{0,1} \approx 0.331$, so in this sense it is no more or less restrictive than the previous standard, Eq. (19). However as the discussion below shows, a larger portion of the twodimensional space of error measures, $\left(\mathcal{E}_{0}, \mathcal{E}_{1}\right)$, is allowed using this new standard. So in this sense it is strictly less restrictive than the detection accuracy standard given in Eq. (19)
To help understand the relationship between these various new standards, Fig. 5 illustrates the regions in the twodimensional space of error measures, $\left(\mathcal{E}_{0}, \mathcal{E}_{1}\right)$, allowed by each version. The original time-domain standard, Eq. (10) with $C_{0} \approx 0.016$, restricts $\mathcal{E}_{0}$ to the region left of the vertical dashed line in this figure. The simplest version of the new standard for detection, the $k=1$ version of Eq. (16) with $C_{1} \approx 0.239$, restricts $\mathcal{E}_{1}$ to the region below the dotted horizontal line. A slightly more complicated standard, Eq. (19) with $C_{0}=C_{1}=0.331$, restricts both $\mathcal{E}_{0}$ and $\mathcal{E}_{1}$ to the region inside the solid-line square. The more complicated compound time-domain standards presented in this Appendix restrict both $\mathcal{E}_{0}$ and $\mathcal{E}_{1}$ to the region inside an ellipse defined in Eq. (B8). The simplest of these uses the compound error measure $\mathcal{E}_{0,1}(\sigma)$ defined in Eq. (B5) with $\sigma=0.5$. This limits points to the region inside the dash-dotted circle defined by $C_{0,1}(0.5) \approx 0.314$. The least restrictive standard of this type uses the error measure $\mathcal{E}_{0,1}(0.284)$ with $C_{0,1}(0.284) \approx 0.331$. It limits points to the region inside the dash-double-dotted ellipse shown in this figure.

Each of these versions of the waveform accuracy standard for detection is sufficient to guarantee the original frequency-domain standards are satisfied. So any point, $\left(\mathcal{E}_{0}, \mathcal{E}_{1}\right)$, in the two-dimensional space of waveform errors, allowed by any of these conditions is an acceptable standard satisfying point. The most general accuracy standard of this type could be obtained by taking the union of the allowed regions inside each of the ellipses defined by Eq. (B8) with $C_{0,1}(\sigma)=\min _{M} C_{0,1}(\sigma, M)$. The boundary of this region qualitatively has the shape of a hyperbola that asymptotes to the vertical dashed line and the horizontal dotted line in Fig. 5. This region is rather complicated to specify exactly, however, so this most general version of these compound standards is probably impractical for widespread use.

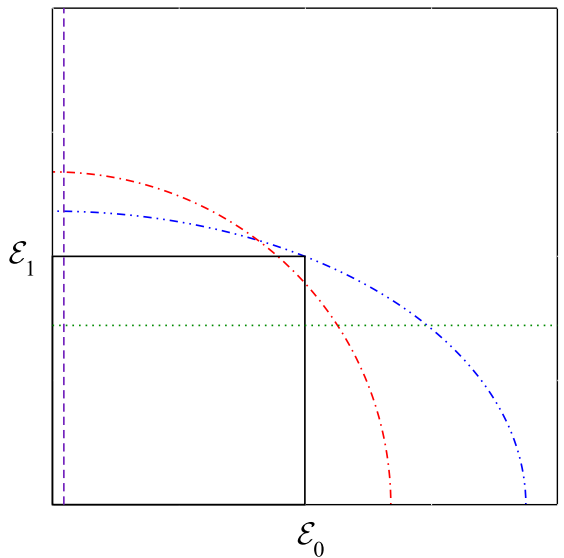

FIG. 5 (color online). Illustrates the allowed regions of the two-dimensional space of model-waveform errors, $\left(\mathcal{E}_{0}, \mathcal{E}_{1}\right)$, satisfying various versions of the accuracy standards discussed in the text. 
[1] L. Blanchet, Living Rev. Relativity 9, 4 (2006).

[2] J. M. Centrella, J. G. Baker, B. J. Kelly, and J. R. van Meter, Annu. Rev. Nucl. Part. Sci. 60, 75 (2010).

[3] B. Abbott et al. (LIGO Scientific Collaboration), Rep. Prog. Phys. 72, 076901 (2009).

[4] F. Acernese et al. (Virgo Collaboration), Classical Quantum Gravity 25, 114045 (2008).

[5] O. Jennrich, Classical Quantum Gravity 26, 153001 (2009).

[6] K. Kuroda (LCGT Collaboration), Classical Quantum Gravity 27, 084004 (2010).

[7] E. E. Flanagan and S. A. Hughes, Phys. Rev. D 57, 4566 (1998).

[8] M. A. Miller, Phys. Rev. D 71, 104016 (2005).

[9] L. Lindblom, B. J. Owen, and D. A. Brown, Phys. Rev. D 78, 124020 (2008).

[10] L. Lindblom, Phys. Rev. D 80, 042005 (2009).

[11] L. Lindblom, Phys. Rev. D 80, 064019 (2009).

[12] D. J. A. McKechan, C. Robinson, and B. S. Sathyaprakash, Classical Quantum Gravity 27, 084020 (2010).

[13] T. Damour, B. R. Iyer, and B. S. Sathyaprakash, Phys. Rev. D 62, 084036 (2000).

[14] M. Giles and E. Süli, Acta Numer. 11, 145 (2002).
[15] J. G. Baker, S. T. McWilliams, J.R. van Meter, J. Centrella, D.-I. Choi, B. J. Kelly, and M. Koppitz, Phys. Rev. D 75, 124024 (2007).

[16] S. Husa, J. A. González, M. Hannam, B. Brügmann, and U. Sperhake, Classical Quantum Gravity 25, 105006 (2008).

[17] M. Boyle, A. Buonanno, L. E. Kidder, A. H. Mroué, Y. Pan, H. P. Pfeiffer, and M. A. Scheel, Phys. Rev. D 78, 104020 (2008).

[18] I. Hinder, F. Herrmann, P. Laguna, and D. Shoemaker, Phys. Rev. D 82, 024033 (2010).

[19] M. Campanelli, C. O. Lousto, H. Nakano, and Yosef Zlochower, Phys. Rev. D 79, 084010 (2009).

[20] T. Chu, H. P. Pfeiffer, and M. A. Scheel, Phys. Rev. D 80, 124051 (2009).

[21] L. Santamaria et al., Phys. Rev. D 82, 064016 (2010).

[22] S. T. McWilliams, B. J. Kelly, and J. G. Baker, Phys. Rev. D 82, 024014 (2010).

[23] D. Shoemaker, LIGO Document T0900288-v3, 2009, http://dcc.ligo.org.

[24] M. A. Scheel, M. Boyle, T. Chu, L.E. Kidder, K. D. Matthews, and H.P. Pfeiffer, Phys. Rev. D 79, 024003 (2009).

[25] M. Boyle, D. A. Brown, and L. Pekowsky, Classical Quantum Gravity 26, 114006 (2009). 